\title{
Long non-coding RNA AL355711 promotes smooth muscle cell migration through the ABCG1/MMP3 pathway
}

\author{
CHUN-MIN KANG ${ }^{1,2}$, WEI-KANG LI ${ }^{1}$, KE-WEI YU ${ }^{1}$, XUE-HENG LI $^{3}$, RUI-YING HUANG ${ }^{3}$, \\ PEI-FENG KE ${ }^{1}$, XING JIN ${ }^{1}$, SHUN-WANG CAO ${ }^{1}$, YING-SHI YUAN ${ }^{1}$, HENG WANG $^{1}$, \\ JUN YAN $^{1}$, WEI-YE CHEN ${ }^{1}$, XIAN-ZHANG HUANG ${ }^{1,2}$ and JING-JING ZHAO ${ }^{3}$ \\ ${ }^{1}$ Department of Laboratory Medicine, The Second Affiliated Hospital of Guangzhou University of Chinese Medicine; \\ ${ }^{2}$ Department of Laboratory Medicine, Guangdong Provincial Hospital of Chinese Medicine, Guangzhou, \\ Guangdong 510120; ${ }^{3}$ Department of Laboratory Medicine, Nanfang Hospital, \\ Southern Medical University, Guangzhou, Guangdong 510515, P.R. China
}

Received July 13, 2021; Accepted September 21, 2021

DOI: $10.3892 / \mathrm{ijmm} .2021 .5040$

\begin{abstract}
Atherosclerosis and related cardiovascular diseases pose severe threats to human health worldwide. There is evidence to suggest that at least $50 \%$ of foam cells in atheromas are derived from vascular smooth muscle cells (VSMCs); the first step in this process involves migration to human atherosclerotic lesions. Long non-coding RNAs (lncRNAs) have been found to play significant roles in diverse biological processes. The present study aimed to investigate the role of lncRNAs in VSMCs. The expression of IncRNAs or mRNAs was detected using reverse transcription-quantitative polymerase chain reaction. The Gene Expression Omnibus datasets in the NCBI portal were searched using the key words 'Atherosclerosis AND tissue AND Homo sapiens' and the GSE12288 dataset. Gene expression in circulating leukocytes was measured to identify patients with coronary artery disease (CAD) or controls, and used to analyze the correlation coefficient and expression profiles. The protein level of ATP-binding cassette sub-family G member 1 (ABCG1) and matrix metalloproteinase (MMP) 3 was determined using immunohistochemistry and western blot analysis. The analysis of mouse aortic roots was performed using Masson's
\end{abstract}

Correspondence to: Dr Xian-Zhang Huang, Department of Laboratory Medicine, The Second Affiliated Hospital of Guangzhou University of Chinese Medicine, 111 Dade Road, Guangzhou, Guangdong 510120, P.R. China

E-mail: huangxz020@163.com

Dr Jing-Jing Zhao, Department of Laboratory Medicine, Nanfang Hospital, Southern Medical University, 1838 Guangzhou Avenue North, Guangzhou, Guangdong 510515, P.R. China

E-mail: 493564616@qq.com

Key words: atherosclerosis, long non-coding RNA AL355711, Vascular smooth muscle cell migration, ATP-binding cassette sub-family G member 1, matrix metalloproteinase 3 and Oil Red O staining. The expression of IncRNA AL355711, ABCG1 and MMP3 was found to be higher in human atherosclerotic plaques or in patients with atherosclerotic CAD. The correlation analysis revealed that ABCG1 may be involved in the regulation between IncRNA AL355711 and MMP3 in atherosclerotic CAD. The knockdown of IncRNA AL355711 inhibited ABCG1 transcription and smooth muscle cell migration. In addition, lncRNA AL355711 was found to regulate MMP3 expression through the ABCG1 pathway. The expression of ABCG1 and MMP3 was found to be high in an animal model of atherosclerosis. The results indicated that lncRNA AL355711 promoted VSMC migration and atherosclerosis partly via the ABCG1/MMP3 pathway. On the whole, the present study demonstrates that the inhibition of IncRNA AL355711 may serve as a novel therapeutic target for atherosclerosis. lncRNA AL355711 in circulating leukocytes may be a novel biomarker for atherosclerotic CAD.

\section{Introduction}

Cardiovascular disease (CVD) is the leading cause of mortality in China (1). As has been reported, $>17$ million individuals succumbed to CVD in 2015, representing $31 \%$ of all global deaths (2). Atherosclerosis, the formation of fibrofatty lesions in the artery wall, causes CVD (2). A deeper understanding of the pathogenesis of atherosclerosis is required in order to develop effective and timely strategies to combat the challenges of CVD (3). However, the pathogenesis of atherosclerosis is complex and is not yet fully understood. The initiation and progression of atherosclerosis involve the pathophysiological activation of multiple cell types, such as vascular endothelial cells, monocytes and vascular smooth muscle cells (VSMCs). During the evolution of atherosclerotic plaques, VSMCs produce matrix metalloproteinases (MMPs) that degrade interstitial collagen, leading to the migration of medial VSMCs into the intima and to the formation of foam cells (2). VSMCs play an important regulatory role in atherosclerosis and plaque development (4).

Emerging evidence has suggested that atherosclerosis is an epigenetic disease involving the interplay of multiple 
epigenetic mechanisms (5). The long non-coding RNA (lncRNA) macrophage-associated atherosclerosis sequence was previously identified and characterized as an essential regulator of macrophage apoptosis, efferocytosis and plaque necrosis in the atherosclerotic plaques of $L d l r^{-/-}$mice via direct interaction with the RNA-binding protein, HuR (6). As previously demonstrated, the lncRNA-fatty acid 2-hydroxylase (FA2H-2)/mixed lineage kinase domain-like protein (MLKL) pathway is essential for regulating the autophagic flux and inflammation through mTOR-dependent signaling, and lncRNA-FA2H-2 knockdown promotes atherosclerosis (7). LincRNA-p21 was previously identified as a novel regulator of neointima formation, VSMC proliferation, apoptosis and atherosclerosis by enhancing p53 activity (8). Smooth muscle cell-enriched lncRNA (SMILR) has been shown to promote VSMC proliferation via the direct regulation of mitotic progression, which may be a potential SMILR-targeting intervention to limit atherogenesis (9). Although a considerable number of molecules have been shown to participate in the regulation of VSMCs in atherosclerosis, numerous others have yet to be discovered.

The present study reported a lncRNA AL355711, a lncRNA that has not been previously reported. It was found that IncRNA AL355711 promoted VSMC migration and atherogenesis. In brief, the present study found that: i) lncRNA AL355711 was overexpressed in human plaques; ii) lncRNA AL355711 knockdown inhibited smooth muscle cell migration in a scratch assay; iii) lncRNA AL355711 upregulated ATP-binding cassette sub-family G member 1 (ABCG1) transcription; iv) lncRNA AL355711 regulated MMP3 expression through the ABCG1 pathway; and v) ABCG1 and MMP3 were highly expressed in an animal model of atherosclerosis.

\section{Materials and methods}

Patients and specimens. A total of five normal and 17 atherosclerotic frozen plaque tissues were randomly collected with informed consent from patients who initially underwent carotid endarterectomy or abdominal aorta surgery at Nanfang Hospital, Southern Medical University (Guangzhou, China) (10,11). Ethical consent was obtained from the Committee for Ethical Review of Research Involving Human Subjects of Southern Medical University (approval no. NFEC-2017-122). Informed consent written was obtained from all the participants.

Cells and culture parameters. Human VSMCs were purchased from the American Type Culture Collection (ATCC CRL-1999) and maintained in Dulbecco's modified Eagle's medium (Gibco; Thermo Fisher Scientific, Inc.) supplemented with $10 \%$ fetal bovine serum (Gibco; Thermo Fisher Scientific, Inc.) and antibiotics (penicillin, $100 \mathrm{U} / \mathrm{ml}$; streptomycin, $100 \mathrm{mg} / \mathrm{ml}$. Gibco; Thermo Fisher Scientific, Inc) at $37^{\circ} \mathrm{C}$ in a humidified incubator containing $5 \% \mathrm{CO}_{2}$. The VSMC cell line is referenced as T/G HA-VSMC in Cellosaurus. (https://web.expasy.org/cellosaurus/CVCL_4009).

Microarray analysis. The microarray analysis was carried out using the Agilent Array platform (Affymetrix U133A chips, Agilent Technologies, Inc). The assay was performed as previously described (12). The expression microarray data can be found at NCBI GEO (Accession no. GSE97210; https://www. ncbi.nlm.nih.gov/geo/query/acc.cgi?acc=GSE97210).

Lentiviral construction and cell transfection. Short hairpin RNA (shRNA) was prepared as previously described (11). The shRNAs were constructed using a lentivirus vector (pHBLVU6-MCS-CMV-ZsGreen-PGK-PURO). Briefly, 293T cells (ATCC CRL-3216) were transfected with a combination of lncRNA AL355711 shRNA, $10 \mu \mathrm{g}$ lentiviral packaging plasmids pSPAX2 and $5 \mu \mathrm{g}$ helper plasmids pMD2G using Lipofiter $^{\mathrm{TM}}$ (Han Bio Co. Ltd.) at $37^{\circ} \mathrm{C}, 5 \% \mathrm{CO}_{2}$ for $72 \mathrm{~h}$. The virus was subsequently harvested by ultracentrifugation at $4^{\circ} \mathrm{C}, 2,000 \mathrm{x} \mathrm{g}$ for $10 \mathrm{~min}$ and at $4^{\circ} \mathrm{C}, 82,700 \mathrm{xg}$ for $120 \mathrm{~min}$. The human VSMCs were cultured in 6-well plates for $12 \mathrm{~h}$ to $50-70 \%$ confluence prior to use. The cells were transfected with lentiviral vectors using polybrene reagent (Santa Cruz Biotechnology, Inc.) in Opti-MEM (Gibco; Thermo Fisher Scientific, Inc.) following the manufacturer's protocols at a multiplicity of infection of 10. Stable knockdown cell clones were generated after 2 weeks using puromycin, and the lncRNA AL355711 level was evaluated using reverse transcriptionquantitative polymerase chain reaction (RT-qPCR). The sequences of sh-AL355711 are presented in Table SI.

Transfection with short interfering RNAs (siRNAs). The cells were transfected with siRNAs specific for ABCG1 protein and scrambled siRNA (Guangzhou RiboBio Co., Ltd.) using Lipofectamine $3000{ }^{\circledR}$ reagent (Invitrogen; Thermo Fisher Scientific, Inc.) following the manufacturer's protocols. The siRNA sequences are presented in Table I. VSMCs $\left(5 \times 10^{4}\right.$ cells/well) were seeded into the wells of 6 -well plates ( $2 \times 10^{6}$ cells/well) and cultured for $12 \mathrm{~h}$ to $50-70 \%$ confluence prior to use. The cells were incubated in Opti-MEM (Gibco; Thermo Fisher Scientific, Inc.) containing $2 \mu \mathrm{l}$ Lipofectamine $3000^{\circledR}$ reagent and $5 \mu \mathrm{l}$ siRNA $(50 \mathrm{nM})$ or siN-Ctrl for $6 \mathrm{~h}$. The supernatant was discarded, and $2 \mathrm{ml}$ complete medium were added to each well. The transfected cells were grown for $48 \mathrm{~h}$ prior to analysis.

RNA isolation and RT-qPCR. RNA isolation and RT-qPCR were performed as previously described (13). Total RNA was extracted from the cultured cells using TRIzol ${ }^{\circledR}$ reagent (Invitrogen; Thermo Fisher Scientific, Inc.). First-strand complementary DNA (cDNA) of lncRNA AL355711 was synthesized using M-MLV Reverse Transcriptase (Promega Corporation), while cDNA was synthesized from total mRNAs using the PrimeScript RT reagent kit (Takara Bio, Inc.), in $20 \mu \mathrm{l}$ reaction volumes containing $2 \mu \mathrm{g}$ total RNA. qPCR was carried out using a SYBR-Green PCR kit (Takara Bio, Inc.) on a Roche Z480 Real-Time PCR system (Roche Molecular Diagnostics). U6 and glyceraldehyde 3-phosphate dehydrogenase (GAPDH) were used as internal controls for lncRNAs and mRNAs, respectively. PCR was performed according to the product instruction with standard thermocycling conditions $\left(50^{\circ} \mathrm{C} 2 \mathrm{~min}, 95^{\circ} \mathrm{C} 2 \mathrm{~min}\right.$, and then 35 cycles of $95^{\circ} \mathrm{C} 15 \mathrm{sec}, 60^{\circ} \mathrm{C} 32 \mathrm{sec}$, and melting curves of $95^{\circ} \mathrm{C} 1 \mathrm{~min}$, $60^{\circ} \mathrm{C} 30 \mathrm{sec}$, and then $\left.40^{\circ} \mathrm{C} 30 \mathrm{sec}\right)$. The expression levels were quantified using the $2^{-\Delta \Delta \mathrm{Cq}}$ method (14). All samples were prepared in triplicate, and the mean value was used for 
comparative analyses. The sequences of the primers used are presented in Table SI.

Cell migration and scratch assay. The cell migration assay was performed using Boyden chambers consisting of Transwell member filter inserts ( $8 \mu \mathrm{m}$; Corning, Inc.). In brief, $2 \times 10^{5}$ cells/well were seeded in a 24 -well plate. The cells that did not penetrate the filter were wiped away after $12 \mathrm{~h}$ using a cotton swab. The cells on the lower surface of the filter were fixed in 4\% paraformaldehyde (Wuhan Servicebio Technology Co., Ltd.) for $20 \mathrm{~min}$, and washed with PBS (Wuhan Servicebio Technology Co., Ltd.) twice. The cells were then stained using $0.5 \%$ crystal violet (Nanjing KeyGen Biotech Co., Ltd.) for $20 \mathrm{~min}$ at room temperature.

The scratch assay was performed using a 6-well plate. In brief, $5 \times 10^{5}$ cells/well were seeded into a 6 -well plate and scratches were created using a pipet tip after $24 \mathrm{~h}$. The cells were cultured in a complete medium with $2 \%$ FBS, and the scratches were imaged at the 0,8 and $24 \mathrm{~h}$ time points using a BX51 microscope (Olympus Corporation).

Western blot analysis. The cells were harvested and lysed in radioimmunoprecipitation assay buffer (Fdbio Science) containing protease and phosphatase inhibitors and phenylmethanesulfonyl fluoride (Fdbio Science). The protein concentration was determined using an enhanced bicinchoninic acid protein assay kit (Fdbio Science), and proteins were separated on a 12\% sodium dodecyl sulfate-polyacrylamide gel and transferred onto a polyvinylidene difluoride membrane (EMD Millipore). The membranes were then blocked with 5\% bovine serum albumin for $1 \mathrm{~h}$ at room temperature and incubated overnight at $4^{\circ} \mathrm{C}$ with primary antibodies ABCG1 (1:1,000; cat. no. NB400-132SS; Novus Biologicals, LLC) and MMP3 (1:1,000; cat. no. sc-21732, Santa Cruz Biotechnology, Inc.). Following incubation with appropriate horseradish peroxidase-conjugated secondary antibodies (1:3,000; cat. no. 7076; Cell Signaling Technology, Inc., 1:3,000; cat. no. 7074; Cell Signaling Technology, Inc.), immunoreactivity was detected using enhanced chemiluminescence with western blotting substrate (Fdbio Science). Protein expression levels were quantified using Quantity One software (V4.6.2; Bio-Rad Laboratories, Inc.) and normalized to the level of $\beta$-actin (overnight at $4^{\circ} \mathrm{C}, 1: 1,000$; cat. no. 3700 ; Cell Signaling Technology, Inc.).

Bioinformatics analysis. The Gene Expression Omnibus (GEO) datasets in the NCBI portal (ncbi.nlm.nih.gov) were searched using the keywords 'Atherosclerosis AND tissue AND Homo sapiens'. One dataset, GSE12288 [110 samples for coronary artery disease (CAD) and 112 samples for the controls] was found. Gene expression in circulating leukocytes was measured to identify patients with CAD or the controls. In this analysis, $\mathrm{R} 3.63$, $\mathrm{R}$ stats package 3.5 .0 and $\mathrm{R}$ pcaPP package 1.9 .73 (https://cran.r-project.org/bin/windows/base/old/3.5.0/) were used to measure the correlation, and Pearson's correlation analysis was used to calculate the correlation coefficient. R ggplot2 was used to draw the first heatmap. R ggplot2, $\mathrm{R}$ ggExtra and R psych (http://cran.r-project.org) were used to draw the correlation scatter plot, and $\mathrm{R}$ ggplot $2 \mathrm{R}$ beeswarm (http://cran.r-project.org)was used to draw the expression scatter plot. 
Animals. An animal model of atherosclerosis was constructed as previously described (11). In brief, apolipoprotein E (ApoE) knockout $\left(\mathrm{ApoE}^{-/}\right.$) mice with a C57BL/6 background were purchased from Guangdong GemPharmatech Co., Ltd. ( $n=9$, male, 6 weeks of age, weighing $20 \mathrm{~g}$; $6 / \mathrm{JGpt}$-Apoeem1Cd82/Gpt). The mice were kept in a specific pathogen-free environment with free access to food and water with a temperature of $20-26^{\circ} \mathrm{C}$ and a humidity of $40-70 \%$, and a 12-h dark/light cycle. Atherosclerosis was induced using a Western high-fat diet (HFD; Open Source Diet D12108C; Research Diets, Inc.; $40 \%$ fat calories and $1.25 \%$ cholesterol) for 12 weeks. The mice were sacrificed humanely after 12 weeks of being fed the HFD, with no suffering or potential pain and/or distress, and ascending aortas, mouse aortic root tissues were collected for analyses. The death of the mice was verified as follows: A lack of respiration, heartbeat and corneal reflex. In total, 4 mice were used as the controls and 5 for the HFD feeding; the experiments were performed once. All animal experiments conformed to the Guide for the Care and Use of Laboratory Animals published by the US National Institutes of Health (NIH Publication no. 85-23, revised in 1996) and were approved by the Nanfang Hospital Animal Experimental Committee (approval no. 20200830005).

Histological analysis, Oil Red O staining, Masson's staining and immunohistochemical analyses of mouse aortic roots. Histological analysis, Oil Red $\mathrm{O}$ staining and immunohistochemical analyses of the mouse aortic roots were performed as previously described $(10,11)$. The mouse aortic roots were fixed in $4 \%$ paraformaldehyde (Wuhan Servicebio Technology Co., Ltd.) $20 \mathrm{~min}$ at room temperature, and stained for $2 \mathrm{~h}$ using Oil Red O (O0625; Sigma-Aldrich; Merck KGaA), and Masson's stain or hematoxylin and eosin (H\&E) staining according to the manufacturer's instructions (G1346 and G1120; Beijing Solarbio Science \& Technology Co., Ltd.). Formalin-fixed, paraffin-embedded sections (4- $\mu \mathrm{m}$-thick) of mouse aortic roots were incubated with ABCG1 (overnight at $4^{\circ} \mathrm{C}, 1: 1,00$; NB400-132SS, Novus Biologicals, LLC) and MMP3 (overnight at $4^{\circ} \mathrm{C}, 1: 1,00$; sc-21732, Santa Cruz Biotechnology, Inc.) antibodies, and the secondary antibodies using the GTVision $^{\text {TM }}$ Anti-mouse/Anti-rabbit Immunohistochemical Analysis kit (including DAB; HRP conjugates, no dilution, cat. no. GK500710; Gene Tech, Inc.) according to the manufacturer's instructions. Images were acquired using a Leica DM 2500 microscope (Leica Microsystems $\mathrm{GmbH}$ ) and analyzed using ImageJ software (version 150; National Institutes of Health).

Statistical analysis. Data are expressed as the mean \pm standard deviation. Data were analyzed using IBM SPSS 20.0 (IBM Corporation) and GraphPad 8.0 (GraphPad Software, Inc.). Statistically significant differences were calculated using the unpaired two-tailed Student's t-test or one-way ANOVA followed by Tukey's post hoc test. A two-tailed probability value of $\mathrm{P}<0.05$ was considered to indicate a statistically significant difference.

\section{Results}

Expression of lncRNA AL355711 and ABCG1 is higher in human atherosclerotic plaques. Arraystar LncRNA Expression
Microarray V3.0 was used on three paired human atherosclerotic tissues and healthy arterial specimens to discover the abnormal expression of lncRNAs. 1ncRNA AL355711 was found to be one of the most significantly upregulated lncRNAs (4.55-fold increase, $\mathrm{P}=0.000000506894775660708$, Table I). The transcriptional regulation of protein-coding genes by lncRNAs has been reported in the literature. The transcription of IncRNAs is now known to regulate the expression of genes in close genomic proximity (cis-acting regulation) (15). The location of the ABCG1 gene was in close proximity to IncRNA AL355711 (Fig. 1A), and the expression of ABCG1 was also higher in human atherosclerotic tissues (3.02-fold increase, $\mathrm{P}=0.00182682036107604$, Table I). The expression microarray data can be found at NCBI GEO (Accession no. GSE97210; https://www.ncbi.nlm. nih.gov/geo/query/acc.cgi?acc=GSE97210). The expression of lncRNA AL355711 was verified using RT-qPCR in five healthy arterial intima tissues and 20 human atherosclerotic plaques (Fig. 1B). Microarrays that measured the expression of IncRNA AL355711 were screened from the GEO database to obtain an overview of the differential expression of lncRNA AL355711 in atherosclerotic CAD. GSE12288, a microarray from the GPL96 platform, was selected. The expression profile of lncRNA AL355711 in circulating leukocytes was used to identify patients with CAD and was assessed using Affymetrix U133A chips. The expression of lncRNA AL355711 and ABCG1 was significantly increased in patients with atherosclerotic CAD (Fig. 1C and D). Therefore, it was demonstrated that IncRNA AL355711 and ABCG1 may play a potential role in atherosclerosis.

Knockdown of lncRNA AL355711 inhibits ABCG1 transcription. The correlation between lncRNA AL355711 and ABCG1 was first analyzed through bioinformatics analysis. The correlation between the AL355711 and ABCG1 mRNA levels in atherosclerotic CAD was found to be positive (Pearson's R=0.15; $\mathrm{P}=0.027$; <0.05; Fig. 1E). Stable lncRNA AL355711 knockdown VSMCs were then constructed. The shRNA lentiviral vector targeting lncRNA AL355711 was constructed to knock down lncRNA AL355711 in order to clarify the transcriptional regulation of lncRNA AL355711. The expression of IncRNA AL355711 was decreased by $>90 \%$ (Fig. 1F). The knockdown of lncRNA AL355711 also decreased the mRNA and protein levels of ABCG1 (Fig. 1F and G). These results suggested that lncRNA AL355711 inhibited the transcription of ABCG1.

lncRNA AL355711 knockdown inhibits smooth muscle cell migration. Smooth muscle cells contribute to the majority of foam cells in $\mathrm{ApoE}^{-/-}$mouse atherosclerosis (16). Smooth muscle cell migration is the first step of atherosclerosis induced by smooth muscle cells. Loss-of function experiments were performed to evaluate the functional role of lncRNA AL355711 in VSMC migration. Stable lncRNA AL355711 knockdown human VSMCs were established using lentivirus (Fig. 1F). IncRNA AL355711 knockdown significantly inhibited the migration of VSMCs, as shown by the results of Transwell assay (Fig. 2A). Moreover, the results of wound healing assay indicated that the wound width was markedly reduced (Fig. 2B). 
A

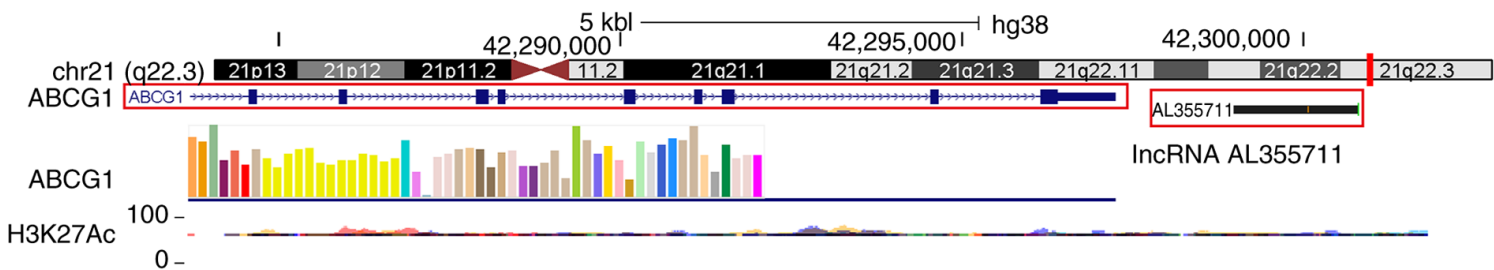

B

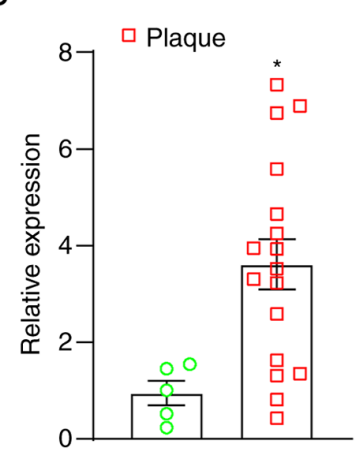

C

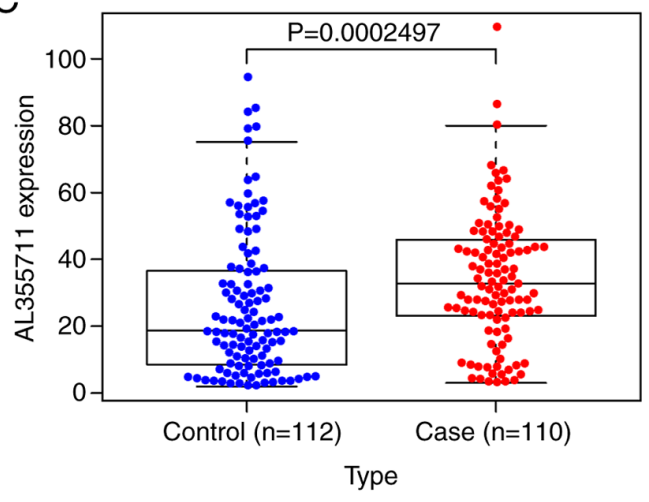

F

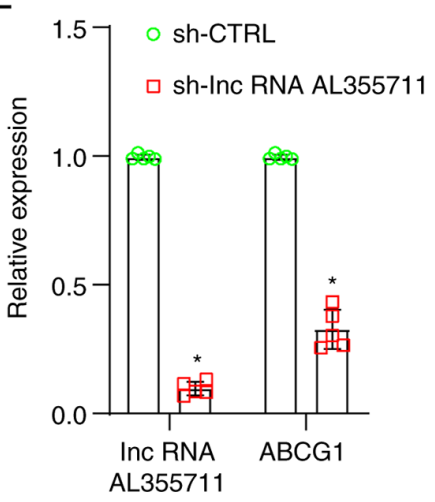

D

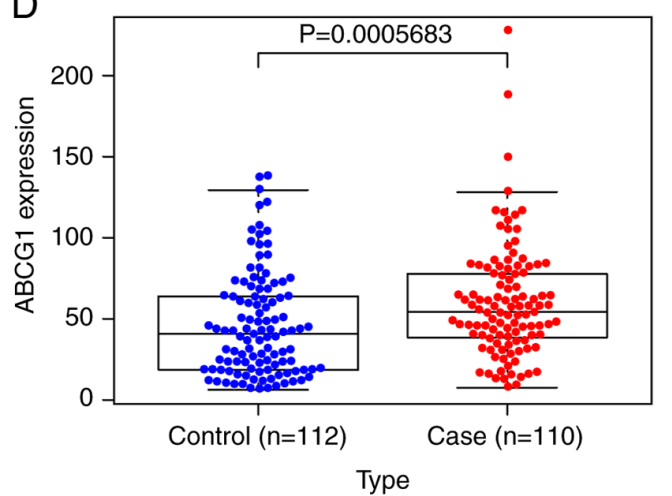

E

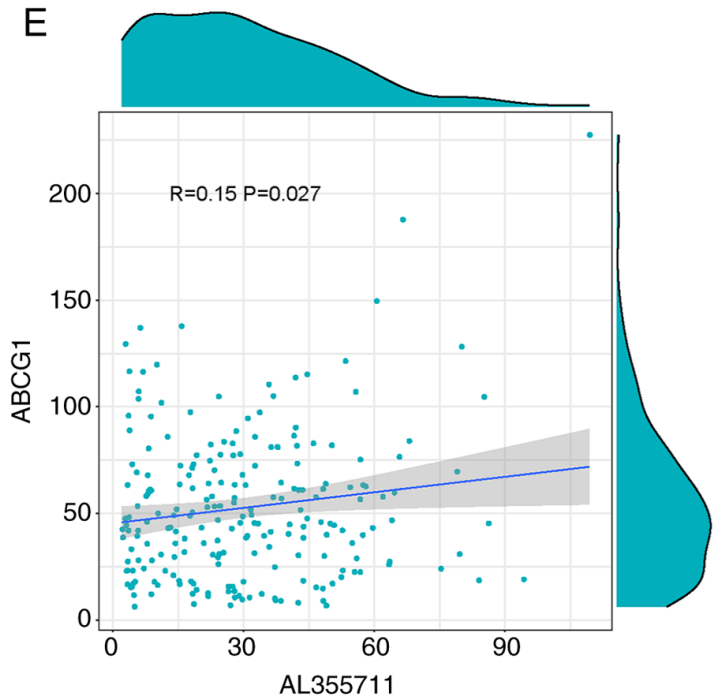

G

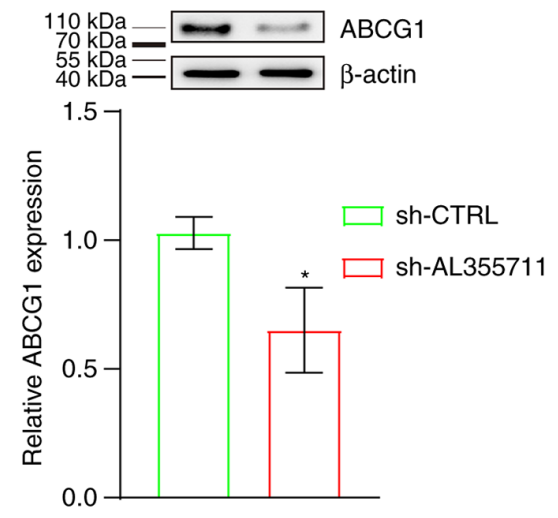

Figure 1. IncRNA AL355711 expression is higher in human atherosclerotic plaques. (A) Positional association between lncRNA AL355711 and ABCG1. (B) Expression of lncRNA AL355711 in plaque tissues was significantly increased. Data shown in graphs are the mean \pm SD. * $\mathrm{P}<0.05$. (C) Expression of IncRNA AL355711 was significantly increased in circulating leukocytes, as detected by bioinformatics analysis in GSE12288 [RNA was extracted from 110 patients with $\mathrm{CAD}(\mathrm{CADi}>23)$ and from 112 partially matched controls without $\mathrm{CAD}(\mathrm{CADi}=0)]$. Gene expression was assessed using Affymetrix U133A chips $(\mathrm{P}=0.0002497$; $<0.001)$. (D) Expression of $\mathrm{ABCG} 1$ was significantly increased in circulating leukocytes, as detected using bioinformatics analysis of the GSE12288 dataset $(\mathrm{P}=0.0005683 ;<0.001)$. (E) Correlation between AL355711 and ABCG1 RNA levels in circulating leukocytes was determined using Pearson's correlation analysis using the GSE12288 dataset (Pearson's $\mathrm{R}=0.15 ; \mathrm{P}=0.027$; $<0.05$ ). (F) lncRNA AL355711 was successfully knocked down, and the mRNA level of ABCG1 decreased significantly. Data shown in the graphs are the mean \pm SD. ${ }^{*}<<0.05$. (G) Protein level of ABCG1 was significantly decreased following lncRNA AL355711 knockdown. Data shown in the graphs are the mean $\pm \mathrm{SD}$. "P<0.05. IncRNA, long non-coding RNA; ABCG1, ATP-binding cassette sub-family G member 1; CAD, coronary artery disease. 
A
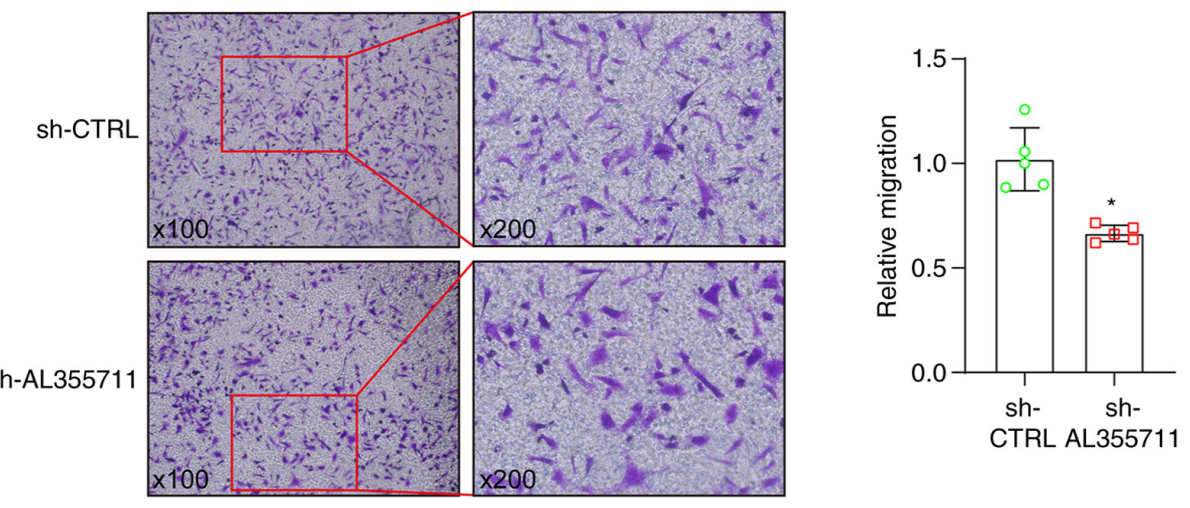

B
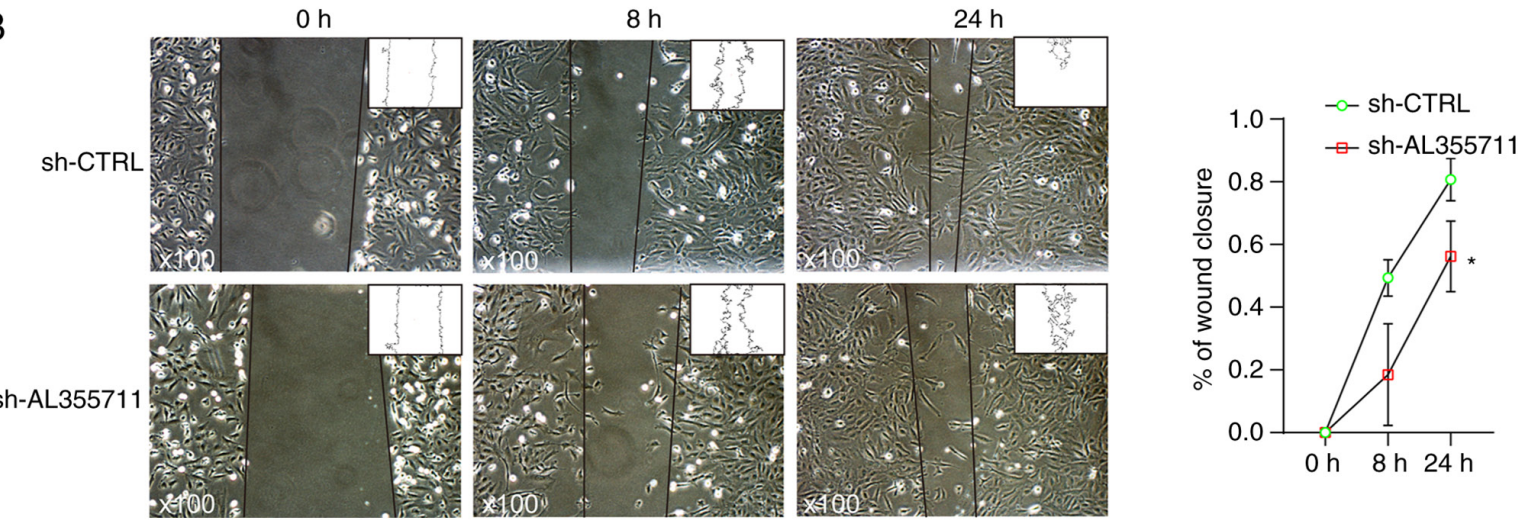

Figure 2. IncRNA AL355711 knockdown inhibits smooth muscle cell migration in the scratch assay. (A) lncRNA AL355711 knockdown inhibited cell migration. The images on the right panel are an enlarge magnification of the red boxed areas of the images in the left panels (left panel, x100 magnification; right panel, x200 magnification). Data shown in the graphs are the mean \pm SD. " $\mathrm{P}<0.05$. (B) lncRNA AL355711 knockdown inhibited cell migration in the scratch assay, reducing wound width and closure (x100 magnification). The inserts in each image illustrate the scratch area. Data shown in the graphs are the mean \pm SD. ${ }^{*} \mathrm{P}<0.05$. IncRNA, long non-coding RNA.

IncRNA AL355711 regulates MMP3 expression through the ABCG1 pathway. The MMP3 level is elevated in patients with fatal and non-fatal cardiovascular outcomes compared with controls. The MMP3 baseline level in patients with a history of CAD is a potential predictor for cardiovascular outcomes (17). The expression of MMPs and matrix-degrading activity has been shown to be increased in vulnerable regions of human atherosclerotic plaques (18). MMP3 knockdown has been shown to attenuate VSMC migration into $t$ scratch wound by $59 \%$ and reduce neointima formation and VSMC accumulation in mouse atherosclerotic plaques, suggesting that MMP3 plays a key role in VSMC migration and neointima formation (19).

The present study performed a bioinformatics analysis of the key migration-associated gene, MMP3, to investigate the mechanisms through which IncRNA AL355711 modulates VSMC migration. The expression of MMP3 was found to be significantly upregulated in patients with atherosclerotic CAD (Fig. 3A). The correlation between AL355711 and MMP3 mRNA levels in atherosclerotic CAD was also found to be positive (Pearson's $\mathrm{R}=0.21 ; \mathrm{P}=0.00002 ; \mathrm{P}<0.05$; Fig. 3B). As aforementioned, IncRNA AL355711 regulated the transcription of ABCG1, and lncRNA AL355711 positively correlated with MMP3; however, correlation between ABCG1 and MMP3 remained unclear. Thus, the correlation between ABCG1 and MMP3 was further analyzed. A positive correlation was found between ABCG1 and MMP3 (Pearson's R=0.32; $\mathrm{P}=0.000000012 ; \mathrm{P}<0.05$; Fig. 3C). Hence, it was suggested that ABCG1 is involved in the regulation between lncRNA AL355711 and MMP3 in atherosclerotic CAD (Fig. 3D).

A specific knockdown probe targeting ABCG1 was then constructed to determine the role of MMP3 in the regulation of VSMC migration. The transfection of si-ABCG1 was successful; the decreased target expression was evidenced using western blot analysis, and si-ABCG1-1 was used in subsequent experiments as it was the most efficient (Fig. 3E). The expression of MMP3 was decreased following lncRNA AL355711 knockdown, and this decrease was partially made more prominent by the knockdown of ABCG1 (Fig. 3F).

ABCG1 and MMP3 are highly expressed in an animal model of atherosclerosis. To the best of our knowledge, no homologous sequence of IncRNA AL355711 exists in mice. Hence, it is impossible to construct a lncRNA AL355711 knockout mouse model. In the present study, a mouse model of atherosclerosis was constructed by feeding male $\mathrm{ApoE}^{-1-}$ mice with a Western HFD for 12 weeks to further verify the role of ABCG1 and MMP3 in atherosclerotic formation. The staining of the aortic roots with Oil Red O (Fig. 4A) and the aortic root cross-sections stained with H\&E (Fig. 4B) indicated that the mouse model of atherosclerosis was successfully constructed using the HFD. The collagen fiber content was significantly decreased in the atherosclerotic plaques (Fig. 4C). The expression of ABCG1 was found to be significantly increased in atherosclerotic plaques (Fig. 4D). 
A

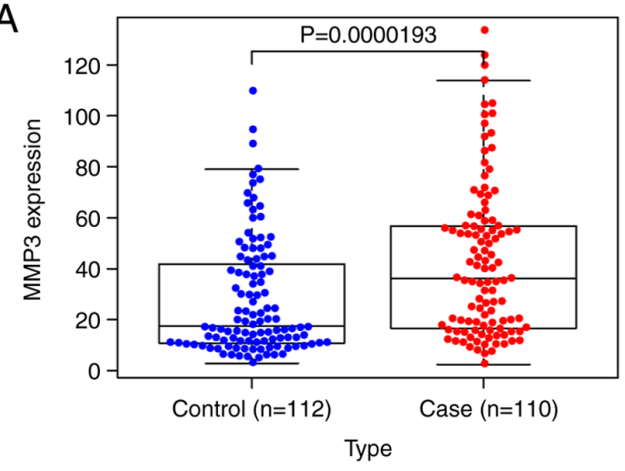

B
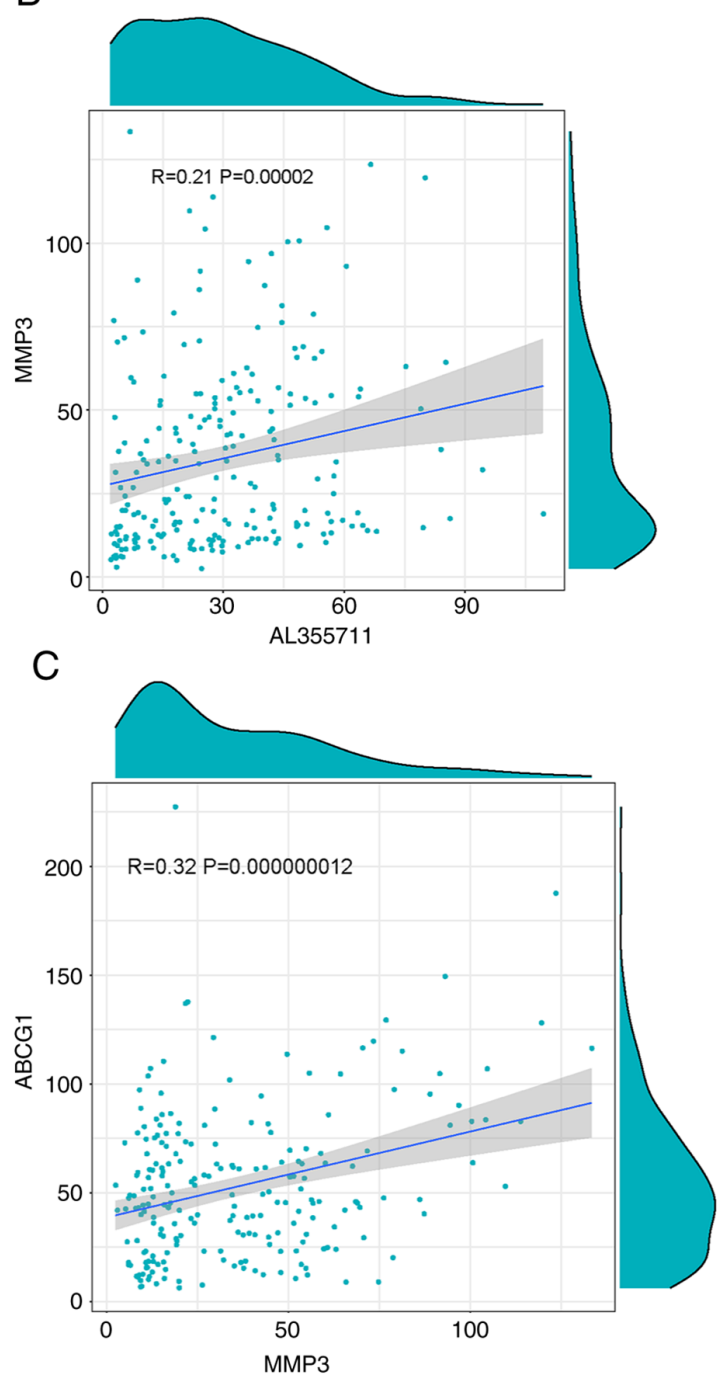

D

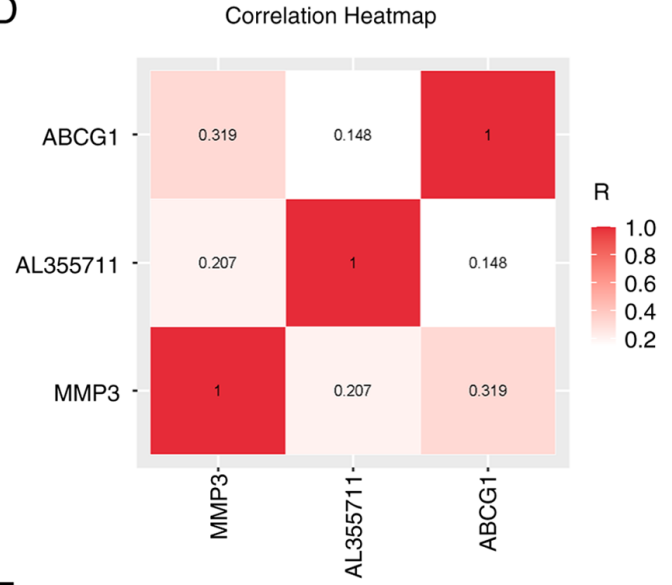

E
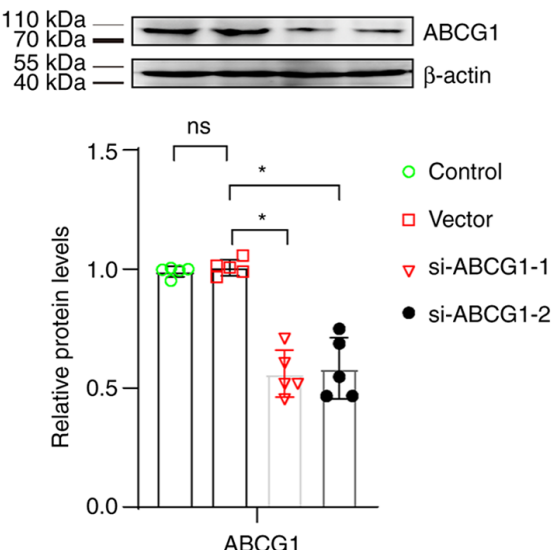

$\mathrm{F}$

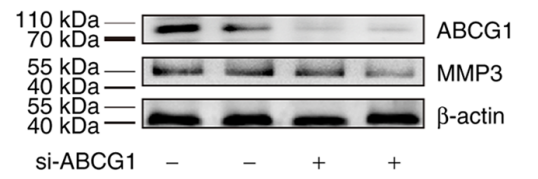

Sh-AL355711 - $\quad+\quad+\quad+$

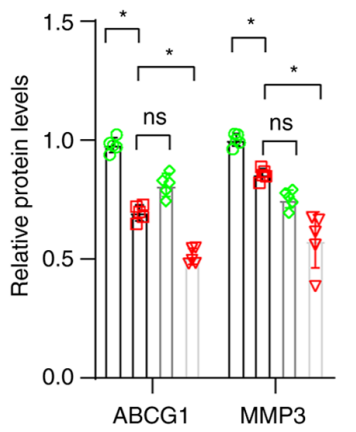

Figure 3. lncRNA AL355711 regulates MMP3 expression through the ABCG1 pathway. (A) Expression of MMP3 was significantly increased, as detected using bioinformatics analysis of the GSE12288 dataset. $\mathrm{P}=0.0000193$; $<0.001$. (B) Correlation between AL355711 and MMP3 RNA levels in circulating leukocytes identifies patients with coronary artery disease using Pearson's correlation analysis of the GSE12288 dataset ( $P$ earson's $\mathrm{R}=0.21 ; \mathrm{P}=0.00002 ;<0.05$ ). (C) Correlation between ABCG1 and MMP3 mRNA levels in circulating leukocytes, as detected using Pearson's correlation analysis of the GSE12288 dataset (Pearson's R=0.32; P=0.000000012; <0.05). (D) Correlation heatmap of AL355711, ABCG1 and MMP3 RNA levels in circulating leukocytes was drawn using Pearson's correlation analysis of the GSE12288 dataset. (E) Transfection with si-ABCG1 was successful, and the scrambled siRNA negative control had no effect on ABCG1 expression. Data shown in the graphs are the mean \pm SD. " $\mathrm{P}<0.05$; ns, indicates no significant differences. (F) Knockdown of ABCG1 enhanced the downregulation of ABCG1 by lncRNA AL355711. Data shown in the graphs are the mean $\pm \mathrm{SD}$. ${ }^{*} \mathrm{P}<0.05$; ns, indicates no significant differences. lncRNA, long non-coding RNA; ABCG1, ATP-binding cassette sub-family G member 1; MMP3, matrix metalloproteinase 3.

The consecutive paraffin-embedded tissue sections were also stained for MMP3, which was found to be highly expressed in atherosclerotic plaques (Fig. 4E). Both MMP3 and ABCG1 were also found to be highly expressed in the same sections of atherosclerotic plaques (Fig. 4F). A schematic diagram of the possible mechanisms through which lncRNA AL355711 promotes VSMC migration and atherogenesis via ABCG1/MMP3 is presented in Fig. 5. 

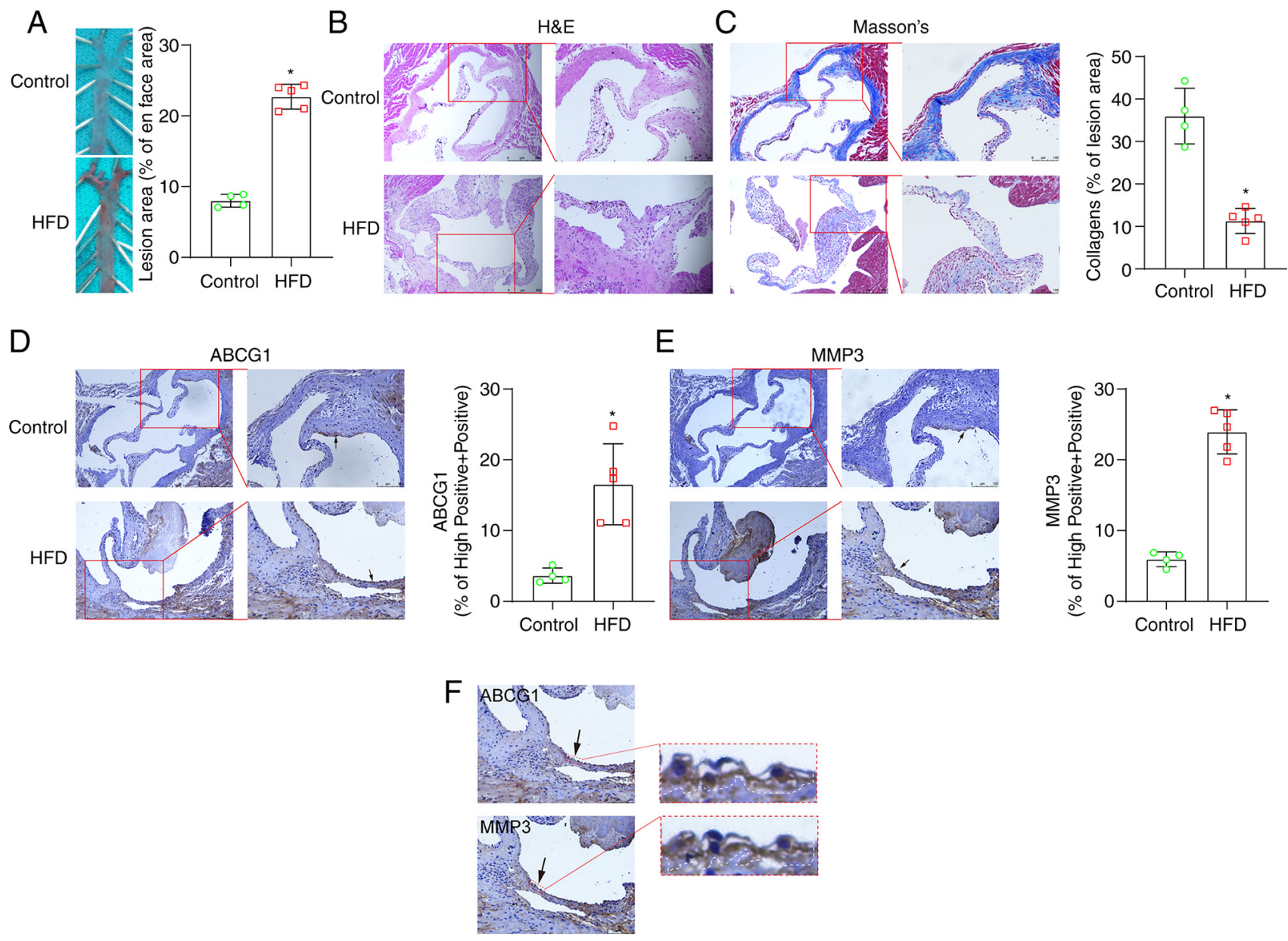

Figure 4. ABCG1 and MMP3 are highly expressed in an animal model of atherosclerosis. An animal model of atherosclerosis was successfully constructed by feeding 6-week-old apolipoprotein E knockout mice with a Western HFD for 12 weeks. (A) Representative en face images of the ascending aorta stained with Oil Red O (x2.5 magnification). A blue mouse pad was used as the supporting platform for the ascending aortas (hence the green-like background). Data shown in the graphs are mean $\pm \mathrm{SD}$. ${ }^{*} \mathrm{P}<0.05$. In total, 4 mice were used as the controls and 5 mice were used in the HFD group. The experiments were performed once. (B) Aortic root cross-sections stained with H\&E. Scale bar left panel, $250 \mu \mathrm{m}$ (x100 magnification); scale bar right panel, $100 \mu \mathrm{m}$ (x200 magnification), The images on the right panel are an enlarge magnification of the red boxed areas of the images in the left panels. (C) Aortic root cross-sections stained with Masson's stain indicated that the HFD diet significantly reduced the collagen fiber content. Data shown in the graphs are the mean \pm SD. "P $<0.05$. Scale bar left panel, $250 \mu \mathrm{m}$ (x100 magnification); scale bar right panel, $100 \mu \mathrm{m}$ (x200 magnification). The images on the right panel are an enlarge magnification of the red boxed areas of the images in the left panels. (D) ABCG1 immunohistochemical staining revealed that the HFD significantly increased the ABCG1 levels in plaques. Data shown in the graphs are the mean $\pm \mathrm{SD}$. ${ }^{*} \mathrm{P}<0.05$. Scale bar left panel, $250 \mu \mathrm{m}$ (x100 magnification); scale bar right panel, $100 \mu \mathrm{m}$ (x200 magnification). The images on the right panel are an enlarge magnification of the red boxed areas of the images in the left panels. (E) MMP3 immunohistochemical staining revealed that the HFD significantly increased the level of ABCG1 in plaques. Data shown in the graphs are the mean \pm SD. *P<0.05. Scale bar left panel, $250 \mu \mathrm{m}$ (x100 magnification); scale bar right panel, $100 \mu \mathrm{m}$ (x200 magnification). The images on the right panel are an enlarge magnification of the red boxed areas of the images in the left panels. (F) MMP3 immunohistochemical staining revealed that the HFD significantly increased the level of ABCG1 in plaques. Data shown in the graphs are the mean $\pm \mathrm{SD}$. ${ }^{*} \mathrm{P}<0.05$. The same paraffin-embedded tissue sections were stained for MMP3 and ABCG1, both of which were highly expressed in the same section of atherosclerotic plaques. Scale bar left panel, $100 \mu \mathrm{m}$ (x200 magnification); arrows indicate the red boxed areas, which are presented as an enlarged magnification in the images on the right (x20,000 magnification). IncRNA, long non-coding RNA; ABCG1, ATP-binding cassette sub-family G member 1; MMP3, matrix metalloproteinase 3; HFD, high-fat diet; H\&E, hematoxylin and eosin.

\section{Discussion}

The morbidity and mortality rates are higher in patients with CVD. Its major clinical manifestations include ischemic heart disease, ischemic stroke and peripheral artery disease (20). Atherosclerosis, the major cause of CVD, involves a complex progression of chronic inflammation, response to injury and disordered lipid metabolism (21). The mechanisms of action of VSMCs in various stages of native atherosclerotic plaque formation vary (22). VSMCs are the most abundant cells in human atherosclerotic lesions and it has been suggested that at least $50 \%$ foam cells in atheroma are derived from VSMCs (16). One of the key findings of the present study was that an uncharacterized lncRNA AL355711 promoted VSMC migration and atherogenesis via ABCG1/MMP3 (Fig. 5).

A number of major modifiable risk factors for atherosclerosis have been identified, including, but not limited to, smoking, obesity, hypertension, hyperlipidemia, hyperglycemia and physical inactivity (1). Non-coding RNAs have been demonstrated to play crucial roles in gene regulation and atherosclerosis (10). IncRNA NEXN-AS1 has been shown to mitigate atherosclerosis by regulating the actin-binding protein NEXN (11). Another study demonstrated that lncRNA HCG11 regulates the proliferation and apoptosis of VSMCs by targeting the miR-144-3p/FOXF1 axis in atherosclerosis (23). lncRNA growth arrest specific 5 (GAS5) has 


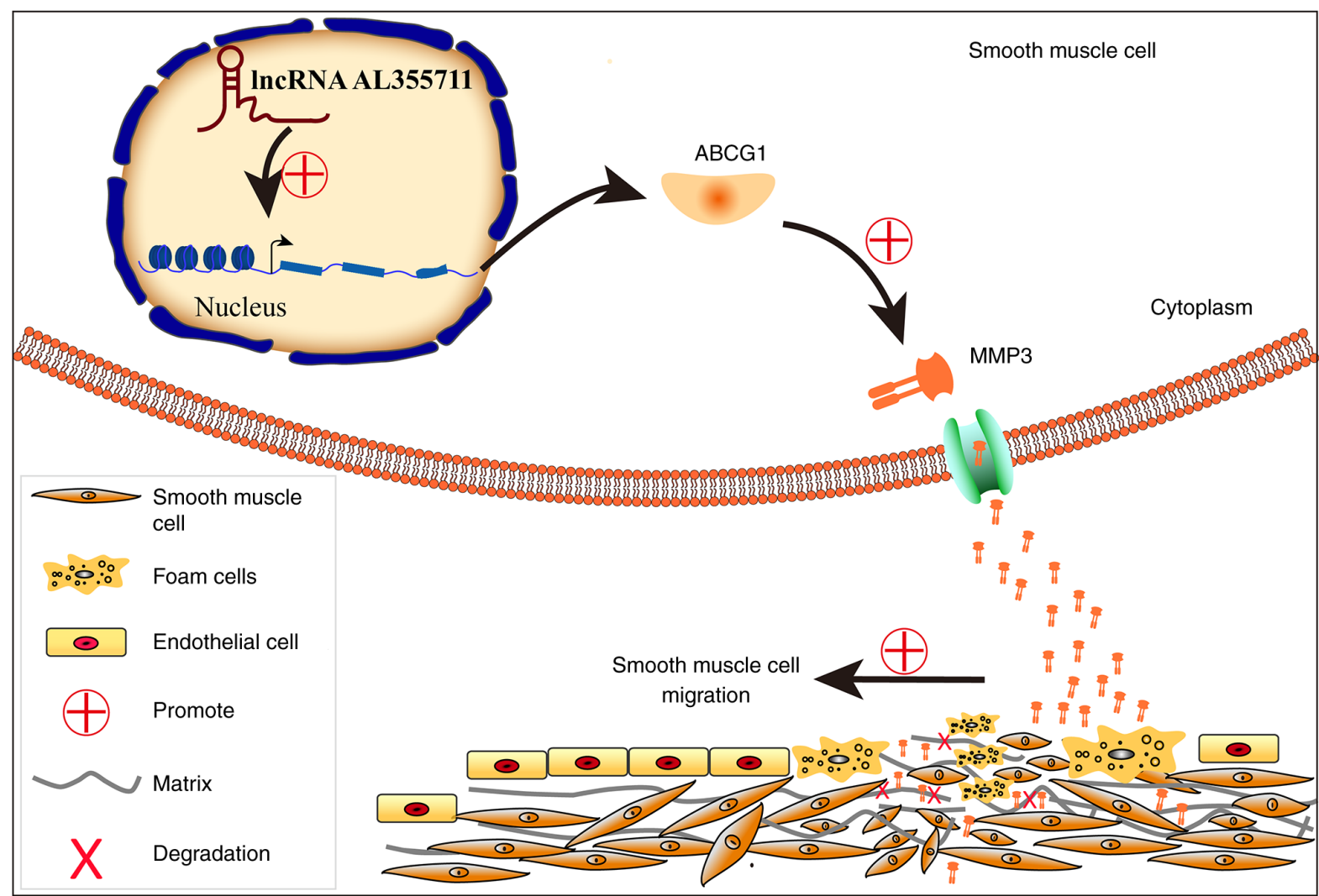

Figure 5. Schematic diagram depicting the role of IncRNA AL355711 in the regulation of smooth muscle cell migration. 1ncRNA AL355711 promoted the transcription of ABCG1, which promoted the protein expression of MMP3, degraded the extracellular matrix, and promoted smooth muscle cell migration. lncRNA, long non-coding RNA; ABCG1, ATP-binding cassette sub-family G member 1; MMP3, matrix metalloproteinase 3.

been found to induce VSMC cycle arrest and apoptosis via the p53 pathway (24). A previous study demonstrated that the knockdown of PVT1 enhanced aortic cell apoptosis, elevated the MMP2 and MMP9 levels, reduced the tissue inhibitor matrix metalloproteinase 1 levels, and increased the levels of pro-inflammatory cytokines (25). At least $50 \%$ of foam cells in atheroma are derived from VSMCs, and the migration of VSMCs is the first step in the formation of a foam cell (22). Hence, the role of lncRNAs in VSMC migration warrants further investigation. The present study identified a novel lncRNA, AL355711, located on chromosome 21q22 in close proximity to the protein-coding gene, ABCG1. IncRNA AL355711 was found to be overexpressed in plaques and positively regulated the expression of ABCG1. IncRNA AL355711 promoted the migration of VSMCs by regulating MMP3 expression via the ABCG1 pathway. IncRNA AL355711 in circulating leukocytes may thus be a novel biomarker for atherosclerosis. IncRNAs are an important class of regulatory molecules; previous studies have demonstrated that lncRNAs may be used as therapeutic targets (26-28). RNA-based therapies, including RNA molecules as drugs and small molecules targeted by RNA, provide a unique opportunity to expand the range of therapeutic targets. However, advancements made in ribonucleic acid therapy are likely to rely on improvements in the design and utilization of appropriate ribonucleic acid molecules, delivery systems, and targeting technologies to ensure efficacy and avoid or minimize nontargeting effects and immunogenicity (29). For the future directions of research, the regulation of the increase in the expression of lncRNA AL355711 remains unclear. In addition, the mechanisms underlying the upregulation of ABCG1 by IncRNA AL355711 are poorly understood. Therefore, the effects of lncRNA AL355711 on atherosclerosis need to be examined in future studies.

MMPs have been implicated in the development and progression of atherosclerosis due to their ability to induce the focal destruction of the vascular extracellular matrix (30). Matrix degradation is a hallmark of high-risk atherosclerosis that leads to myocardial infarction and stroke (31). MMP1, MMP3 and MMP12 are positively associated with cardiovascular and cerebrovascular events in patients with carotid atherosclerosis (32). MMPs play important roles in VSMC migration (33). MMP3 is a key member of the MMP family. It is present in coronary atherosclerosis (34). The serum levels of MMP1, MMP3, MMP7 and MMP12 have also been shown to be elevated in patients with chronic obstructive pulmonary disease and carotid plaques in the carotid artery (35). MMP3 haplotype 2 has been found to be associated with a reduced risk of myocardial infarction and an increased risk of hemorrhagic stroke; thus, the MMP3 haplotype may predict both cardiac events and stroke (36). MMP3 is required for IL-1-induced VSMC invasion (37). The present study discovered a novel regulatory role for MMP3. IncRNA AL355711 increased the level of MMP3 by upregulating ABCG1 expression. The expression of MMP3 and ABCG1 increased in atherosclerotic plaques in mice. The present study found that MMP3 and ABCG1 were upregulated in circulating leukocytes of atherosclerotic CVD. The expression of MMP3 and ABCG1 
in circulating leukocytes may be a novel biomarker for atherosclerotic CVD. It was found that ABCG1 expression positively correlated with MMP3 expression, and ABCG1 knockdown decreased the protein level of MMP3. In addition, MMP3 and ABCG1 were highly expressed in atherosclerotic plaques in mice, and both MMP3 and ABCG1 were highly expressed in the same section of atherosclerotic plaques. The small sample size of the mouse model used may be a limitation of the present study. The authors aim to overcome this limitation in future studies. The present study identified novel modulating pathways of MMP3 and validated these in a mouse model of atherosclerotic plaques. However, the mechanisms underlying the upregulation of MMP3 by lncRNA AL355711 and ABCG1 remain unclear.

Pursuing the biological implications of the emerging genetic results represents a promising area for future investigation. In conclusion, the present study identified a previously uncharacterized lncRNA, AL355711, that promotes VSMC migration and atherogenesis via ABCG1/MMP3. These findings may enhance the current understanding of the pathogenesis of atherosclerosis and indicate that IncRNA AL355711, ABCG1 and MMP3 are potential biomarkers and therapeutic targets for atherosclerotic CVD.

\section{Acknowledgements}

Not applicable.

\section{Funding}

The present study was supported by grants from the National Natural Sciences Foundation of China (grant no. 82072336), the Natural Science Fund of Guangdong (grant nos. 2019A1515010178 and 2019B1515120004), the Science and Technology Program of Guangzhou (grant nos. 202002020038, 202102010173 and 202103000025), the Medical Scientific Research Foundation of Guangdong Province of China (grant nos. A2019282 and A2020108) and the Project of Administration of Traditional Chinese Medicine of Guangdong Province of China (grant no. 20211180).

\section{Availability of data and materials}

The datasets used and/or analyzed in this study are available from the corresponding author on reasonable request.

\section{Authors' contributions}

XZH and JJZ designed the study. CMK designed the experiments. CMK, KWY, WKL and PFK performed the experiments (CMK and KWY, performed the cell migration scratch assay; WKL performed RT-qPCR and western bot analysis; PFK performed the bioinformatics analysis). CMK performed the in vivo experiments. JJZ contributed the patient samples. XHL and RYH contributed to the immunohistochemical analysis. SWC performed the pathological analyses (histological analysis, Oil Red O staining, Masson's staining, hematoxylin and eosin staining). CMK wrote the manuscript. JY, WYC, YSY, HW and XJ contributed to the cell migration and scratch analysis. CMK and JJZ confirm the authenticity of all the raw data. All authors have read and approved the final manuscript.

\section{Ethics approval and consent to participate}

The present study was approved by the Ethics Committee of Nanfang HospitalNFEC-2017-122. Informed consent written was obtained from all the participants. All animal experiments conformed to the Guide for the Care and Use of Laboratory Animals published by the US National Institutes of Health (NIH Publication no. 85-23, revised in 1996) and were approved by the Nanfang Hospital Animal Experimental Committee (approval no. 20200830005).

\section{Patient consent for publication}

Not applicable.

\section{Competing interests}

The authors declare that they have no competing interests.

\section{References}

1. Zhao D, Liu J, Wang M, Zhang X and Zhou M: Epidemiology of cardiovascular disease in China: Current features and implications. Nat Rev Cardiol 16: 203-212, 2019.

2. Libby P, Buring JE, Badimon L, Hansson GK, Deanfield J, Bittencourt MS, Tokgözoğlu L and Lewis EF: Atherosclerosis. Nat Rev Dis Primers 5: 56, 2019.

3. Vasan RS and Benjamin EJ: The future of cardiovascular epidemiology. Circulation 133: 2626-2633, 2016.

4. Pan H, Xue C, Auerbach BJ, Fan J, Bashore AC, Cui J, Yang DY, Trignano SB, Liu W, Shi J, et al: Single-cell genomics reveals a novel cell state during smooth muscle cell phenotypic switching and potential therapeutic targets for atherosclerosis in mouse and human. Circulation 142: 2060-2075, 2020.

5. Xu S, Kamato D, Little PJ, Nakagawa S, Pelisek J and Jin ZG: Targeting epigenetics and non-coding RNAs in atherosclerosis: From mechanisms to therapeutics. Pharmacol Ther 196: 15-43, 2019.

6. Simion V, Zhou H, Haemmig S, Pierce JB, Mendes S, Tesmenitsky Y, Pérez-Cremades D, Lee JF, Chen AF, Ronda $\mathrm{N}$, et al: A macrophage-specific lncRNA regulates apoptosis and atherosclerosis by tethering HuR in the nucleus. Nat Commun 11: 6135, 2020.

7. Guo FX, Wu Q, Li P, Zheng L, Ye S, Dai XY, Kang CM, Lu JB, $\mathrm{Xu} \mathrm{BM}, \mathrm{Xu}$ YJ, et al: The role of the LncRNA-FA2H-2-MLKL pathway in atherosclerosis by regulation of autophagy flux and inflammation through mTOR-dependent signaling. Cell Death Differ 26: 1670-1687, 2019.

8. Wu G, Cai J, Han Y, Chen J, Huang ZP, Chen C, Cai Y, Huang H, Yang Y, Liu Y, et al: LincRNA-p21 regulates neointima formation, vascular smooth muscle cell proliferation, apoptosis, and atherosclerosis by enhancing p53 activity. Circulation 130: 1452-1465, 2014.

9. Mahmoud AD, Ballantyne MD, Miscianinov V, Pinel K, Hung J, Scanlon JP, Iyinikkel J, Kaczynski J, Tavares AS, Bradshaw AC, et al: The human-specific and smooth muscle cell-enriched LncRNA SMILR promotes proliferation by regulating mitotic CENPF mRNA and drives cell-cycle progression which can be targeted to limit vascular remodeling. Circ Res 125: 535-551, 2019.

10. Dong XH, Lu ZF, Kang CM, Li XH, Haworth KE, Ma X, Lu JB, Liu XH, Fang FC, Wang CS, et al: The long noncoding RNA RP11-728F11.4 promotes atherosclerosis. Arterioscler Thromb Vasc Biol 41: 1191-1204, 2021.

11. Hu YW, Guo FX, Xu YJ, Li P, Lu ZF, McVey DG, Zheng L, Wang Q, Ye JH, Kang CM, et al: Long noncoding RNA NEXN-AS1 mitigates atherosclerosis by regulating the actin-binding protein NEXN. J Clin Invest 129: 1115-1128, 2019. 
12. Bai HL, Lu ZF, Zhao JJ, Ma X, Li XH, Xu H, Wu SG, Kang CM, $\mathrm{Lu} \mathrm{JB}, \mathrm{Xu} \mathrm{YJ}$, et al: Microarray profiling analysis and validation of novel long noncoding RNAs and mRNAs as potential biomarkers and their functions in atherosclerosis. Physiol Genomics 51: 644-656, 2019.

13. Kang CM, Bai HL, Li XH, Huang RY, Zhao JJ, Dai XY, Zheng L, Qiu YR, Hu YW and Wang Q: The binding of lncRNA RP11-732M18.3 with 14-3-3 $\beta / \alpha$ accelerates p21 degradation and promotes glioma growth. EBioMedicine 45: 58-69, 2019.

14. Livak KJ and Schmittgen TD: Analysis of relative gene expression data using real-time quantitative PCR and the 2(-Delta Delta C(T)) method. Methods 25: 402-408, 2001.

15. Ponting CP, Oliver PL and Reik W: Evolution and functions of long noncoding RNAs. Cell 136: 629-641, 2009.

16. Wang Y, Dubland JA, Allahverdian S, Asonye E, Sahin B, Jaw JE, Sin DD, Seidman MA, Leeper NJ and Francis GA: Smooth muscle cells contribute the majority of foam cells in ApoE (Apolipoprotein E)-deficient mouse atherosclerosis. Arterioscler Thromb Vasc Biol 39: 876-887, 2019.

17. Guizani I, Zidi W, Zayani Y, Boudiche S, Hadj-Taieb S, Sanhaji H, Zaroui A, Mechmeche R, Mourali MS, Feki M and Allal-Elasmi M: Matrix metalloproteinase-3 predicts clinical cardiovascular outcomes in patients with coronary artery disease: A 5 years cohort study. Mol Biol Rep 46: 4699-4707, 2019.

18. Galis ZS, Sukhova GK, Lark MW and Libby P: Increased expression of matrix metalloproteinases and matrix degrading activity in vulnerable regions of human atherosclerotic plaques. J Clin Invest 94: 2493-2503, 1994.

19. Johnson JL, Dwivedi A, Somerville M, George SJ and Newby AC: Matrix metalloproteinase (MMP)-3 activates MMP-9 mediated vascular smooth muscle cell migration and neointima formation in mice. Arterioscler Thromb Vasc Biol 31: e35-e44, 2011.

20. Du X, Patel A, Anderson CS, Dong J and Ma C: Epidemiology of cardiovascular disease in China and opportunities for improvement: JACC international. J Am Coll Cardiol 73: 3135-3147, 2019

21. Libby P, Bornfeldt KE and Tall AR: Atherosclerosis: Successes, surprises, and future challenges. Circ Res 118: 531-534, 2016.

22. Allahverdian S, Chaabane C, Boukais K, Francis GA and Bochaton-Piallat ML: Smooth muscle cell fate and plasticity in atherosclerosis. Cardiovasc Res 114: 540-550, 2018.

23. Liu Y, Cui X, Wang C and Zhao S: LncRNA HCG11 regulates proliferation and apoptosis of vascular smooth muscle cell through targeting miR-144-3p/FOXF1 axis in atherosclerosis. Biol Res 53: 44, 2020

24. Tang R, Mei X, Wang YC, Cui XB, Zhang G, Li W and Chen SY: LncRNA GAS5 regulates vascular smooth muscle cell cycle arrest and apoptosis via 533 pathway. Biochim Biophys Acta Mol Basis Dis 1865: 2516-2525, 2019.

25. Zhang Z, Zou G, Chen X, Lu W, Liu J, Zhai S and Qiao G: Knockdown of lncRNA PVT1 inhibits vascular smooth muscle cell apoptosis and extracellular matrix disruption in a murine abdominal aortic aneurysm model. Mol Cells 42: 218-227, 2019.
26. Subramaniam N, Nair R and Marsden PA: Epigenetic regulation of the vascular endothelium by angiogenic LncRNAs. Front Genet 12: 668313, 2021

27. Wang YQ, Xu ZM, Wang XL, Zheng JK, Du Q, Yang JX and Zhang HC: LncRNA FOXC2-AS1 regulated proliferation and apoptosis of vascular smooth muscle cell through targeting miR-1253/FOXF1 axis in atherosclerosis. Eur Rev Med Pharmacol Sci 24: 3302-3314, 2020.

28. Kumar MM and Goyal R: LncRNA as a therapeutic target for angiogenesis. Curr Top Med Chem 17: 1750-1757, 2017.

29. Yu AM, Choi YH and Tu MJ: RNA drugs and RNA targets for small molecules: Principles, progress, and challenges. Pharmacol Rev 72: 862-898, 2020.

30. Johnson JL: Metalloproteinases in atherosclerosis. Eur J Pharmacol 816: 93-106, 2017.

31. Monaco C, Gregan SM, Navin TJ, Foxwell BM, Davies AH and Feldmann M: Toll-like receptor-2 mediates inflammation and matrix degradation in human atherosclerosis. Circulation 120: 2462-2469, 2009.

32. Hu W, Wei R, Wang L, Lu J, Liu H and Zhang W: Correlations of MMP-1, MMP-3, and MMP-12 with the degree of atherosclerosis, plaque stability and cardiovascular and cerebrovascular events. Exp Ther Med 15: 1994-1998, 2018.

33. Ma L, Zhang L, Wang B, Wei J, Liu J and Zhang L: Berberine inhibits Chlamydia pneumoniae infection-induced vascular smooth muscle cell migration through downregulating MMP3 and MMP9 via PI3K. Eur J Pharmacol 755: 102-109, 2015.

34. Seifi M, Fallah S and Firoozrai M: Influence of genetic polymorphism in matrix metalloproteinase-3 on extent of coronary atherosclerosis and risk of coronary artery stenosis. Arch Med Res 40: 600-604, 2009.

35. Kraen M, Frantz S, Nihlén U, Engström G, Löfdahl CG, Wollmer P and Dencker M: Matrix metalloproteinases in COPD and atherosclerosis with emphasis on the effects of smoking. PLoS One 14: e0211987, 2019.

36. Kaplan RC, Smith NL, Zucker S, Heckbert SR, Rice K and Psaty BM: Matrix metalloproteinase-3 (MMP3) and MMP9 genes and risk of myocardial infarction, ischemic stroke, and hemorrhagic stroke. Atherosclerosis 201: 130-137, 2008.

37. Alexander MR, Moehle CW, Johnson JL, Yang Z, Lee JK, Jackson CL and Owens GK: Genetic inactivation of IL-1 signaling enhances atherosclerotic plaque instability and reduces outward vessel remodeling in advanced atherosclerosis in mice. J Clin Invest 122: 70-79, 2012.

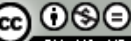

This work is licensed under a Creative Commons Attribution-NonCommercial-NoDerivatives 4.0 International (CC BY-NC-ND 4.0) License. 